\title{
Cost Data Visualisation
}

\author{
Andrew WOOD ${ }^{\mathrm{a}}$, Leigh KIRKWOOD ${ }^{\mathrm{a}, 1}$, Zijin FENG ${ }^{\mathrm{a}}$, Sultan ALHAYDHAL ${ }^{\mathrm{a}}$, \\ Abdullah ALOMRAN ${ }^{\mathrm{a}}$, Rayan BIN TALEB ${ }^{\mathrm{a}}$, Isidro DURAZO-CARDENAS ${ }^{\mathrm{a}}$ and \\ Andrew STARR ${ }^{\mathrm{a}}$ \\ ${ }^{a}$ Cranfield University, College Road, Cranfield, MK43 0AL, UK
}

\begin{abstract}
Decision making using the methodologies and analysis generated by the cost engineering function is widely considered good practice across industry, as a way to support both technical engineering decisions and fundamental business decisions. One persistent challenge for the professional cost engineer is to present cost data and information to decision makers and a mix of audiences. Data visualisation is therefore an important element to ensure that data is presented in a clear, effective and convenient format to ensure sufficient insights can be gathered. This work explores different data presentation and visualisation approaches. This review highlighted this topic as a research gap that this paper is novel in addressing. The review findings are further explored through a series of semi-structured interviews with experts in relevant fields to establish effective data visualisation methods, along with the challenges associated with presenting cost data to a variety of audiences. Chart embellishments are one explored area of potential to increase the engagement and understanding of visualisations.
\end{abstract}

Keywords. Cost-engineering, data visualisation, data.

\section{Introduction}

'Data is just a collection of numbers until you turn it into a story' [1]. Data visualisation involves presenting data in an effective and convenient format that is clear and easy to understand, to assist decision makers in making important judgements and business decisions [2]. The data presentation methods used must include a certain level of complexity to communicate the detail decision makers require. However, to be of use the methods used must be clear enough to ensure sufficient insights can still be gathered.

The challenge area involved in the interface between engineering and business is the area of the cost engineering profession and this interface between disciplines means that there is a mix of audiences to present to, from the very technical to the less technical. Furthermore, there are a variety of reasons for using the analysis generated by the cost engineering function, as they typically support both technical engineering decisions and fundamental business decisions. Despite this challenge there is a lack of literature focusing on the challenges faced in communication of this cost engineering data. Given the importance of cost engineering data and information to support business decisions, any improvement in the clarity of communication can lead to better decision making and improve the financial health of a business.

\footnotetext{
${ }^{1}$ Corresponding Author. 1.kirkwood@cranfield.ac.uk
} 
To get a more detailed understanding of the difficulties in this problem-space, a literature review was completed, and is presented in section 2. The methodology is outlined in section 3 and section 4 involved a series of interviews with experts. Together these activities inform the discussion and conclusions outlined in section 5.

\section{Literature Review}

Visualisation approaches can be categorised as either static or interactive. Static visualisations are usually in the form of diagrams or charts embedded in a larger body of text. In this format, the text conveys the story, and the image typically provides supporting evidence or related details [3]. Static graphs are most appropriate when a snapshot or series of snapshots are able to effectively convey the required information [4]. Each static visualisation method can be further classified based on factors such as: the dimensions of data [5], the number of variables and whether the data is static or dynamic (changing over time).

Interactive data visualisation enables users to interact with graphs and tables in real time to manipulate the data on display [6]. This visualisation approach enables users to go beyond the graphical information offered by static graphs and explore data in greater depths to reveal unseen insights [3]. Interactive visualisations generally allow; data "drill down", data filtering and data sorting (e.g. into ascending or descending order). Haara et al [7] support the use of interactivity by finding that it improves the transparency and controllability of dynamic decision-making processes, enabling appropriate decisions that reflect real time data.

Applying to both static or interactive methods, Duarte [8] recognised the importance of context when presenting and visualising data as well as establishing whether visualisations are required to communicate or declare data and information, or to explore a data set. Kumar [9] notes that because visualisations are utilised in every field in some way, methods used will depend upon the context of the visualisation requirements. Few studies have examined how users choose different visualisation formats to suit their requirements, but a study by Luo [6] goes some way to investigating the impact of different factors on the preferences of visualisation formats.

Infographics (or information graphics), involve combining data and visuals to form a shareable story that is easy to understand [10] and has gained significant traction due to social media. As a communication method, infographics are considered to be one of the most suitable for non-technical audiences [11]. Rininsland et al [12] suggested that infographics may not be considered true data visualisations as they guide audiences through a story where key facts and information are clearly depicted, as opposed to chartbased data visualisations where data is objectively left to be discovered by the audience.

Visual embellishment is a technique where supplementary data information or imagery is added to a graphical visualisation, with the intention of either improving clarity or providing additional information [13]. 


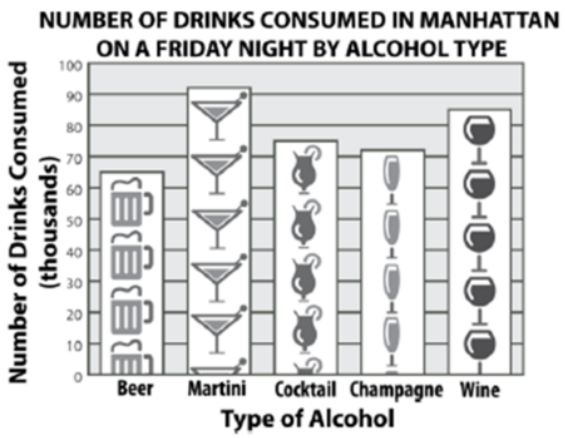

Figure 1. Example visual embellishment that presents customer preferences [13]

Caution is required to avoid what Tufte defined as 'chart junk' which describes any form of embellishment that does not provide any new information to the audience, and argued embellishments 'clutter' graphs and charts and should be removed [14]. Embellishments are a contributing factor to the 'data-ink ratio', a term also proposed by Tufte, in which he argues that all ink not used to present key data should be removed [14]. Alternatively, the study by Bateman et al [13] found that additional embellishments or visual images actually help audiences to remember both the topic and details of a chart and have an impact on how well the data within the chart can be communicated.

During the literature review, no evidence of academic papers that focused on the visualisation of cost data was found. We can conclude therefore that though much effort has been put into the general area of data visualisation and presentation, there remains a more specific research gap with regard to the communication of cost engineering information.

\section{Methodology}

The work presented here proceeded in four phases: project definition, literature review, interviews and data analysis. During the project definition phase the overall aim, objectives and scope were set. The second phase was a literature review that explored data visualisation, the different elements of cost engineering and a variety of specific visualisation methods and approaches. This is a similar methodological approach to the work of Hollberg et al [15] who identified 27 different visualisation methods used to present Life-Cycle Assessment (LCA) results. The literature review revealed a number of research gaps, and identified topics to explore in phase three.

Phase three involved a series of semi-structured interviews, conducted online with experts in relevant fields. These interviews developed a baseline understanding of:

- Presentation requirements of cost data

- Challenges when presenting cost data to a variety of audiences

- Effective data visualisation methods and tools

The interviews therefore follow a similar methodology to Bruce-Hyrkäs et al [16] who examined the current challenges of performing LCA and Life-Cycle Costing calculations in the building sector, based on the results of a survey and interviews. Data analysis was conducted and reported in phase four. 


\section{Results}

$75 \%$ of interview respondents when questioned whether improvements to visualisations were required emphasised the importance and challenge of adapting cost engineering visualisations to the requirements of clients. Several respondents recognised that a common understanding of client expectations is critical to minimise misconceptions and miscommunications.

$25 \%$ of respondents also noted the changing role of visualisations from a fixed to a more dynamic style, whereby different dimensions and depths of data can be evaluated, exploiting the availability of more complex real-time data.

When exploring the challenges of presenting cost data to a variety of audiences, strong evidence was found across the majority of interviews that communication is the greatest challenge faced. Adapting to the background knowledge, capability and the expectations of audiences based on their role are major hurdles to overcome. Further to this, $38 \%$ of interviews identified fundamental cultural, behavioural and intellectual challenges that are also present when presenting cost data. One interview explored how cost estimation is a social phenomenon where "decisions are made not only by leadership but by a democratic culture, where it is essential to find a way of creating a simple story or picture that is recognised and accepted by key stakeholders". Alternatively, from an intellectual perspective, one interview recognised the importance for people to have an "underlying understanding of the data that is used, the influence of different cost drivers and the impact each decision is likely to have on wider cost applications". A second respondent explored the intellectual challenges presented by suggesting that an individual's interest, level of understanding in a task or the ability to express the accuracy of data may impede the effectiveness of tailored cost data visualisations. $25 \%$ of respondents also reported how some of these challenges are restricted by the capability of current 2D static visualisation and presentation methods. To overcome this, suggestions such as chart embellishments (e.g. symbols and other graphical interfaces) to provide different types of information and varying levels of data penetration were put forward by an interview respondent.

When identifying the most effective data visualisation tools, a wide variety of visualisation methods employed in cost applications were reported across all interviews. Conventional static visualisation graphs were commonly used along with more advanced visualisation tools and software. However, there was clear evidence that these methods are tailored to suit specific purposes. Excel was the most commonly used tool for data presentation and visualisation, however some respondents expressed concerns as to whether this method enabled sufficient detail and information to be conveyed to a variety of audiences. Industry respondents also revealed in interviews that specific 'in-house' styles of presenting data are an effective way to communicate consistent data and information across an organisation. Alternatively, when questioned on the future use of visualisation and the way cost data and information is presented, $63 \%$ of respondents outlined the potential value of integrating more interactive visualisations to increase engagement and improve the quality of decisions. The move away from desktop-based visualisations, towards a variety of more dynamic visualisation styles that integrates AI technology and utilises the capabilities of VR/AR to deliver real time insights in greater depth was also proposed by some respondents. 


\section{Discussion and Conclusions}

Improving the visualisation of cost data and information should be an area of greater academic interest. There is also increasing interest in industry to exploit more sophisticated visualisations to drive business decisions as data is becoming significantly more abundant and complex. There has been a lot of work around the general area of data visualisation and presentation, improving the accuracy of estimates and developing costing solutions, however little attention has been paid to the communication of cost engineering information and methods that could improve the way that cost data is presented to audiences. The literature review revealed little academic evidence that focused on the visualisation of cost data. Though graphical examples in cost engineering papers were found, there was little observed consistency in the visualisation methods used to present cost data, nor any justification as to why certain graphical visualisations were used in particular cost applications. The interviews conducted addressed the research gaps identified from the literature review.

The interviews revealed a widespread use of relatively simple static charts to convey cost data and information. The interviews built on the work by Kumar [9] by demonstrating that the visualisation methods adopted should be generally tailored to suit specific purposes and cost applications. This is understandable when recognising that different visualisation methods and techniques can be more/less suited to presenting data in different contexts. Due to the wide-ranging activities and applications, visualisation improvements may not always be necessary; many people have the capability to generate effective static visualisations in Excel that suit their intended applications.

The popularity of simple static charts could be a result of the relatively short time required to prepare conventional visualisations. Companies often adopt their own 'inhouse' style of presenting data, following internally developed templates and 'best practices' to minimise visualisation preparation. Familiarity in presentation or visualisation styles is a key factor in overcoming communication challenges across an organisation, by generating a simple and consistent method that is widely understood and accepted by key stakeholders. Companywide familiarity with cost build-up techniques reduces the frequency of data questioning, increases the confidence in the integrity of the data and therefore reduces the need to invest in more complex visualisation methods.

The literature review and interviews both indicated the potential value of integrating more interactive visualisations in cost applications to reflect more dynamic data, especially considering the gradual shift away from traditional desktop visualisations. Interactivity will undoubtably lead to a richer presentation regarding the insights that can be generated, facilitating faster but more appropriate decisions to be made in future. However, there is a risk that increased interactivity may not always lead to greater understanding. The ability to manipulate data and visualisations by 'drilling down' into specific parameters may result in inconsistencies in the interpretation and understanding of the overall picture of the data. From an audience perspective, is it essential to maintain the contextual element of the cost data and information to avoid assuming that accurate insights will be automatically made by individuals.

The literature review revealed chart embellishments have potential to convey information more effectively than a standard chart [13]. However there is a risk that charts could become 'over-embellished', thereby distracting readers from the data and making graphs more challenging to interpret [14]. Further investigation into the effectiveness of embellishments in cost visualisation is therefore required. The authors 
plan validation of the findings of this paper through a survey. Additional further work could focus on identifying industry specific guidance and recommendations.

The interviews revealed that an awareness of the role and expectations of the audience, along with their technical competencies, is crucial when generating cost data visualisations. Visualisations therefore need to again be adapted and tailored to suit these different requirements of audiences.

When selecting the most appropriate methodologies for data presentation and visualisation in cost challenges, a fine balance needs to be sought between a visualisation that can be understood by a variety of audiences and one that contains a desired level of complexity to ensure sufficient insights can be gathered. In some applications, simple visualisations can have a much greater impact on audiences. Alternatively, overly complex visualisations may be overwhelming and ineffective for other audiences. This paper outlines that this balance should be driven by the requirements of audiences.

\section{References}

[1] N. Martin, 'Data Visualization: How To Tell A Story With Data' (2018). https:/www.forbes.com/sites/nicolemartin1/2018/11/01/data-visualization-how-to-tell-a-story-withdata/?sh=15b730274368 (Last accessed April 2021).

[2] C.P. Killen, J. Geraldi, \& A. Kock., The role of decision makers' use of visualisations in project portfolio decision making, International Journal of Project Management 38 (2020), 267-277.

[3] E. Segel. \& J. Heer., Narrative Visualisation: Telling Stories with Data, IEEE Transactions on Visualisation and Computer Graphics 16 (2010), 1139-1148.

[4] C. Pirrello, 'Effective Visualization Techniques for Data Discovery and Analysis', SAS Global Forum 2010 Conference, (2010), Paper 235.

[5] D. Keim., Information Visualisation and Visual Data Mining, IEEE Transactions on Visualisation and Computer Graphics 8[1] (2002), 1-8.

[6] W. Luo., User choice of interactive data visualisation format: The effects of cognitive style and spatial ability, Decision Support Systems 122 (2019), 1-11.

[7] A. Haara., J. Pykalainen., A. Tolvanen. \& M. Kurttila., Use of interactive data visualisation in multiobjective forest planning, Journal of Environmental Management 210 (2018), 71-86.

[8] N. Duarte, 'The Quick and Dirty on Data Visualisation'. Harvard Business Review (2014). https://hbr.org/2014/04/the-quick-and-dirty-on-data-visualization (Last accessed February 2021).

[9] S. Kumar., A Review of Recent Trends and Issues in Visualisation, International Journal on Computer Science and Engineering 8[3] (2016), 41-54.

[10] E. Walter. \& J. Gioglio., The Power of Visual Storytelling: How to Use Visuals, Videos, and Social Media to Market Your Brand, McGraw-Hill, New York, 2014.

[11] B. Tversky., 'Some ways that maps and graphs communicate' in Spatial Cognition II: Integrating Abstract Theories, Empirical Studies, Formal Methods and Practical Applications, Springer, New York, 2000.

[12] Æ.H. Rininsland, M. Heydt. \& P.N. Castillo., D3.js: Cutting-edge Data Visualisation, Packt, Birmingham, 2017.

[13] S. Bateman, R. Mandryk, C. Gutwin, A. Genest, D. McDine. \& C. Brooks., 'Useful Junk? The effects of visual embellishment on comprehension and memorability of charts', 28th SIGCHI Conference on Human Factors in Computing Systems, (2010), 2573-2582.

[14] E.R. Tufte., The Visual Display of Quantitative Information. Graphics Press, Cheshire, 1983.

[15] A. Hollberg, B. Kiss, M. Röck, B. Soust-Verdaguer, A.H. Wilberg, S. Lasvaux, A. Galimshina. \& G. Habert., Review of visualising LCA results in the design process of buildings, Building and Environment 190 (2021), 1-13.

[16] T. Bruce-Hyrkäs, P. Pasanena. \& R. Castroa., 'Overview of Whole Building Life-Cycle Assessment for Green Building Certification and Ecodesign through Industry Surveys and Interviews', 25th CIRP Life Cycle Engineering (LCE) Conference, (2018), 178-183. 